\title{
Why Astronomy is Useful and Should be Included in the School Curriculum
}

\author{
John R. Percy \\ University of Toronto, Mississauga ON Canada L5L 1 C6 \\ E-mail: jpercy@utm.utoronto.ca
}

Even in my astronomically-developed province of Ontario, Canada, with an excellent public education system, astronomy was not a compulsory topic in the school curriculum until quite recently. So I began collecting a list of reasons why it should be, in the hope of persuading those who set the curriculum.

It would be instructive, however, to start by asking: "Why is astronomy not included in the curriculum?" Here are some possible reasons: (i) Astronomy is perceived to be irrelevant to practical concerns such as health, nutrition, agriculture, environment, engineering, and the economy in general; this is particularly true in developing countries. (ii) Most school teachers have little or no knowledge of astronomy, or astronomy teaching; in fact, they may have the same deeply-rooted misconceptions as their students. (iii) Astronomy is perceived as requiring night-time activities ("the stars come out at night, the students don't"), and expensive and complex equipment such as telescopes. (iv) Astronomy is perceived as being solely "Western" by some non-Western cultures. (v) There may be conflict - real or perceived - between astronomy and personal beliefs such as religion, culture, and pseudo-science; in fact, astronomy is sometimes viewed as being as speculative as pseudo-science. (vi) Many of the available resources are designed for affluent schools in affluent countries, or for different latitudes, longitudes, and languages. (vii) Astronomy may be seen as allied with high technology, with all its real and perceived dangers.

Many of these reasons are based on a lack of an astronomical "tradition" in a country or region. This is one more reason for all members of "the astronomical community" to speak and work together in promoting astronomy.

Now we can address the main topic of this presentation: the reasons why astronomy is useful, and should be part of the school curriculum - in science, or some other place. These can be grouped in several categories:

Cultural and Historical: Astronomy is deeply-rooted in almost every culture, as a result of its practical applications, and its philosophical implications. Among the scientific revolutions of history, astronomy stands out. In the recent lists of "the hundred most influential people of the millennium", a handful of astronomers were always included. Astronomy, by its nature, requires observations from different latitudes and longitudes, and thus fosters international co-operation. It also requires observations over many years, decades, and centuries, thus linking generations and cultures of different times.

Practical: Astronomy has obvious practical applications to time keeping; calendars; daily, seasonal, and long-term changes in weather; navigation; the 
effect of solar radiation; tides; and impacts of asteroids and comets with the earth.

Scientific and Technological: Astronomy is a forefront science which has advanced the physical sciences in general by providing the ultimate physical laboratory - the universe - in which scientists encounter environments far more extreme than anything on earth. It has advanced the geological sciences by providing examples of planets and moons in a variety of environments, with a variety of properties. Astronomical calculations have spurred the development of branches of mathematics such as trigonometry, logarithms, and calculus; now they drive the development of computers: astronomers use a large fraction of all the supercomputer time in the world. Astronomy has led to other technological advances, such as low-noise radio receivers, detectors ranging from photographic emulsions to electronic cameras, and image-processing techniques now used routinely in medicine, remote sensing etc. Its knowledge is essential as human kind enters the era of space exploration.

Aesthetic and Emotional: Astronomy reveals our cosmic roots, and our place in time and space. It deals with the origins of the universe, galaxies, stars, planets, and the atoms and molecules of life - perhaps even life itself. It addresses one of the most fundamental questions of all - are we alone in the universe? Astronomy promotes environmental awareness, through images taken of our fragile planet from space, and through the realization that we may be alone in the universe. Astronomy reveals a universe which is vast, varied, and beautiful - the beauty of the night sky, the spectacle of an eclipse, the excitement of a black hole. Astronomy thus illustrates the fact that science has cultural as well as economic value. It has inspired artists and poets through the ages. Astronomy harnesses curiosity, imagination, and a sense of shared exploration and discovery.

Pedagogical: Astronomy, in the classroom, provides an example of an alternative approach to "the scientific method" - observation, simulation, and theory, in contrast to the usual experiment and theory approach. Astronomy, if properly taught, can promote rational thinking, and an understanding of the nature of science, through examples drawn from the history of science, and from present issues such as pseudo-science. Astronomy can be used to illustrate many concepts of physics, such as gravitation, light, and spectra. Astronomy, by introducing students to the size and age of objects in the universe, gives them experience in thinking more abstractly about scales of time, distance, and size. Astronomy is the ultimate interdisciplinary subject, and "integrative approach" and "cross-curricular connections" are increasingly important concepts in modern school curriculum development.

Societal: Astronomy attracts young people to science and technology, and hence to careers in these fields. Astronomy can promote and increase public awareness, understanding, and appreciation of science and technology, among people of all ages. Astronomy is an enjoyable, inexpensive hobby for millions of people. "The stars belong to everyone!"

Editor's Note: This presentation was followed by an open discussion of the many ways that astronomy can be - and is - included in the school curriculum, worldwide. 